\title{
MULTI-FLUID MODEL FOR SUSPENSION FILTRATION IN POROUS MEDIA: EFFECTS OF PARTICLE TRAPPING AND MOBILIZATION
}

\author{
KRISTINA I. TOLMACHEVA, SERGEI A. BORONIN \& ANDREI A. OSIPTSOV \\ Center for Hydrocarbon Recovery, Skolkovo Institute of Science and Technology, Russia
}

\begin{abstract}
We present the further development of the multi-fluid model of suspension filtration in porous media. The model is derived from conservation laws in the multi-fluid approach, where the three phases, namely the carrier fluid, the suspended particles, and the particles trapped in pores, are treated as three different continua described by hydrodynamic variables. Fluid fluxes through large pores of the porous medium and through narrow pores of the packed bed of deposited particles are explicitly taken into account by introducing two permeabilities. These are the key novel features which distinguish it from the classical deep bed filtration model. The model predictions are compared with laboratory data sets on the contamination of core samples. The most recent progress in the model development is mainly in taking into account particle mobilization and compressibility of the fluid. Applications of the model are primarily in the oil and gas industry: drilling mud invasion and cleanup in the near-wellbore zone, suspension filtration in propped hydraulic fractures, as well as permeability damage and recovery in the near-wellbore zone of injection wells, which are used to maintain reservoir pressure.
\end{abstract}

Keywords: suspension, fines migration, filtration, trapping, mobilization, critical velocity.

\section{INTRODUCTION}

Filtration of suspensions and particle transport in porous media are widely encountered in various natural and technological processes including a number of oilfield applications: the permeability dynamics of a near-wellbore zone during drilling and well cleanup; filtration of particle-laden fluid during hydraulic fracture cleanup and the operation of an injection well. The last one is used to increase the oil recovery in a nearby production wells by injecting a low-viscosity fluid into formation thus maintaining high reservoir pressure. During the filtration of a particle-laden fluid injected into a formation, fine particles can deposit on pore walls and plug the pore throats, which leads to a reduction in the rock permeability [1]. The study of particle capture and entrainment into a flow allows one to minimize the reduction in the well injection rate due to contamination of the surrounding porous medium and optimize the operation of the injection well.

There is a number of models developed earlier for suspension filtration in a porous medium. The most frequently used model is a so-called deep-bed filtration model [2]-[4]. In the framework of this approach, three-continua description of a particle-laden filtration flow is used. The continua are carrier fluid, suspended and trapped particles. The rate of particle trapping is assumed to be proportional to suspended particle concentration, flow velocity and a certain function of trapped particle concentration. An overview of different expressions for the particle trapping rate obtained theoretically and experimentally are presented in [5]. The number of tuning parameters is typically in the range from one to five. While the particles are trapped or deposited in the porous medium, the permeability is altered, which for non-colloidal particles can be described by the power law [4].

The deep-bed filtration model was developed further by Gruesbeck and Collins [6]. The key novel concept is a parallel-pathway approach. The porous medium is represented as the collection of pathways of two types. In pathways of the first type (with small pore radius), 
the particles can only be plugged, while in the pathways of the second type (with large pore size), the particles can deposit on pore walls and can be entrained (or mobilized) into the flow. The expression for the particle mobilization rate was proposed. It was obtained experimentally that there exists a critical velocity of mobilization, below which the mobilization of particles does not occur. Above this velocity, the rate of mobilization is found to be proportional to the concentration of trapped particles and flow velocity [2], [6]. The expressions for particle trapping and mobilization rates contain four tuning parameters, which are proposed to be determined experimentally.

In Rajagopalan and Tien [7], the analytical expression for particle trapping coefficient $\lambda$ is obtained by considering the Happel's cell approach to a porous medium (a spherical collector). It is found that the formula is valid only for sufficiently small particles with the diameter not larger than 0.18 times the diameter of the grains forming the porous medium.

For non-colloidal (i.e. larger than $1 \mu \mathrm{m}$ ) particles, the criteria of particle capture and sticking were proposed in Herzig et al. [2]. These expressions can be used for calculation of probability of particle trapping, but not for determination of the trapping coefficient.

Existing models for filtration of suspensions containing non-colloidal fines involve two tuning parameters for description of the particle mobilization rate and minimum two tuning parameters involved into the expression for the trapping rate.

The aim of the present study is to decrease a number of tuning parameters in expressions for rate of particle trapping and mobilization by taking into account the effect of a finite porosity and permeability of a packed bed formed by the trapped particles.

\section{FORMULATION OF THE PROBLEM}

The multiphase filtration of particle-laden fluids in a porous medium is considered [8]. The new element of our approach is that the trapped fines inside a porous medium form a packed bed through which the carrier fluid is filtered (Fig. 1). A porous medium is represented by two classes of channels: large pore channels and small pore channels. The large channels are formed by the porous matrix with exclusion of trapped particles and a void space between them. The small channels are formed by the gaps between the trapped particles. Fluid can flow both in large and small pore channels, while the suspended particles can be transported in large pore channels only. In order to describe this effect, we introduce two porosities: $\varphi_{c}$ describing the volume fraction of large pore channels, and $\varphi_{t}$ describing the total volume fraction of large and small pore channels:

$$
\varphi_{c}=\varphi_{0}-\frac{\sigma}{C_{\max }}, \varphi_{t}=\varphi_{0}-\sigma
$$

Here, $\varphi_{0}$ is the initial porosity, $C_{\max }$ is the maximum volume concentration of random close packing and $\sigma$ is the volume concentration of trapped particles.

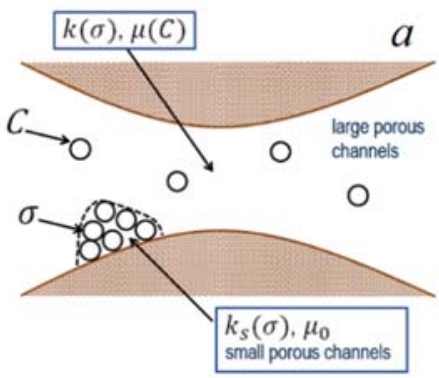

Figure 1: The sketch of the suspension filtration in a porous medium. 
The multiphase filtration of suspension is described using the three-continua approach with different continua being a carrier fluid (with density $\rho_{\gamma}^{f}$ and filtration velocity $u_{\gamma}^{f}$ ), suspended particles (with volume concentration $C$ and filtration velocity $u_{\gamma}^{p}$ ) and trapped particles (with volume concentration $\sigma$ ). Mass balance equations for the continua are shown in eqns (2), (3) and (4):

$$
\begin{gathered}
\frac{\partial}{\partial t}\left[\rho_{\gamma}^{f} s_{\gamma}\left(\varphi_{t}-C \varphi_{c}\right)\right]+\frac{\partial}{\partial x}\left(\rho_{\gamma}^{f} u_{\gamma}^{f}\right)=0, \gamma=1,2 \\
\frac{\partial}{\partial t}\left[\varphi_{c} s_{\gamma} C\right]+\frac{\partial}{\partial x} u_{\gamma}^{p}=-q_{\gamma}, \gamma=1,2 \\
\frac{\partial \sigma}{\partial t}=q_{\gamma}, \quad \gamma=1,2
\end{gathered}
$$

Here, $s_{\gamma}$ is the phase saturation and $q_{\gamma}$ is the rate of particle trapping and mobilization.

Filtration velocities $u_{\gamma}^{f}$ and $u_{\gamma}^{p}$ of the carrier fluid and particles are expressed in terms of the suspension filtration velocity in large pore channels $u_{\gamma}$ and carrier fluid filtration velocity in small pore channels $u_{s}$ by taking into account the volume fraction of suspended particles $C$ as shown in eqns (5) and (6):

$$
\begin{gathered}
u_{\gamma}=-\frac{k k_{\gamma}}{\mu_{\gamma}} \frac{\partial p}{\partial x}, u_{s}=-\frac{k_{s} k_{\gamma}}{\mu_{\gamma, 0}} \frac{\partial p}{\partial x} \\
u_{\gamma}^{p}=C u_{\gamma}, u_{\gamma}^{f}=(1-C) u_{\gamma}+u_{s}
\end{gathered}
$$

Here, $k$ is the permeability of the porous medium, $k_{\gamma}$ is the relative permeability, $\mu_{\gamma}$ is the suspension viscosity, $\mu_{\gamma, 0}$ is the carrier fluid viscosity, $p$ is the fluid pressure. [9]:

The permeability of small pore channels in the case of total trapping $k_{s, 0}$ is defined after

Here, $d_{p}^{2}$ is particle diameter.

$$
k_{s, 0}=\frac{\left(1-C_{\max }\right) d_{p}^{2}}{180 C_{\max }^{2}}
$$

The permeabilities of large and small pore channels are defined as functions of trapped particle concentration after [3], [4]:

$$
k=k_{0}\left(1-\frac{\sigma}{\varphi_{0} C_{\max }}\right)^{3}, k_{s}=k_{s 0}\left(\frac{\sigma}{\varphi_{0} C_{\max }}\right)^{3} .
$$

The suspension viscosity is defined as the function of concentration of suspended particles $C[10]$ :

$$
\mu_{\gamma}=\mu_{\gamma, 0}\left(1-\frac{C}{C_{\max }}\right)^{-1,89}
$$

In the present study, we consider trapping of fines only due to their plugging in pore channels. We neglect the dependence of the particle trapping $q_{t, \gamma}$ on the concentration of trapped particles, so that the expression contains a single tuning parameter $\lambda$ :

$$
q_{t, \gamma}=C u_{\gamma} \lambda \text {. }
$$

The rate of particle mobilization in a porous medium $q_{m, \gamma}$ is described after [6]:

$$
q_{m, \gamma}=\alpha \sigma\left(u_{\gamma}-u_{c r i t, \gamma}\right) \Theta\left(u_{\gamma}-u_{c r i t, \gamma}\right) .
$$

Here, $\alpha$ is the mobilization coefficient, which is to be tuned against the experimental data, $u_{c r i t, \gamma}$ is the critical velocity of mobilization, which can be determined theoretically.

\subsection{Critical velocity of mobilization}

For determination of critical velocity of mobilization, we consider the problem of entrainment of a spherical particle from the plane surface into a laminar flow. Forces acting 
on the particle are as follows: the buoyancy force $F_{b}$, the adhesion $F_{a}$ [11], the friction $F_{f}$ and the drag $F_{d}[12]$, [13]:

$$
\begin{gathered}
F_{b}=\frac{\pi}{6} \Delta \rho g d_{p}^{3} . \\
F_{a}=c_{1} d_{p}, c_{1}=O\left(10^{-5}\right) . \\
F_{d}=1.7 \cdot 6 \pi \mu d_{p}{ }^{2} \frac{u_{\gamma}}{R} .
\end{gathered}
$$

Here, $R$ is the tube radius. We assume that the friction force is proportional to the net vertical force acting on a particle so that following relation holds:

$$
F_{f}=\kappa\left(F_{a}+F_{b}\right)=\kappa\left(c_{1} d_{p}+\frac{\pi}{6} \Delta \rho g d_{p}^{3}\right)
$$

In what follows we assume that $\kappa=1$. The critical velocity of particle mobilization is determined according to the force balance:

$$
\begin{gathered}
F_{d}=F_{f} . \\
u_{c r i t}=\frac{R}{6 \cdot 1.7 \mu}\left(\frac{c_{1}}{\pi} \frac{1}{d_{p}}+\frac{\Delta \rho g}{6} d_{p}\right) .
\end{gathered}
$$

\section{NUMERICAL IMPLEMENTATION AND VALIDATION}

The numerical solution is carried out using a finite-difference approach and a uniform staggered grid. Details of the numerical algorithm can be found in Boronin et al. [8].

For validation of the expressions for the critical velocity (17), we calculated the value of critical velocity for the flow conditions of experiment described in Gruesbeck and Collins [6]. The particle-free fluid was injected into contaminated porous sample and the concentration of suspended particles in the effluent is measured. We obtained a good agreement between the value of mobilization velocity determined via expression (17) and the experimental value reported in Gruesbeck and Collins [6] $(0.448 \mathrm{~cm} / \mathrm{s}$ and $0.58 \mathrm{~cm} / \mathrm{s}$, respectively). We carried out numerical simulations of the experiment [6]. Good agreement between the numerical results and the experimental data, is obtained when both trapping and mobilization of fines are taken into account. At the first stage of the filtration, the velocity of $0.75 \mathrm{~cm} / \mathrm{s}$ is maintained and the trapping and mobilization coefficients were tuned by minimization of the discrepancy between the numerical results and the experiment. Then the second stage of the filtration is simulated with the same tuning parameters and good agreement between the simulations and the experimental data is obtained (Fig. 2).

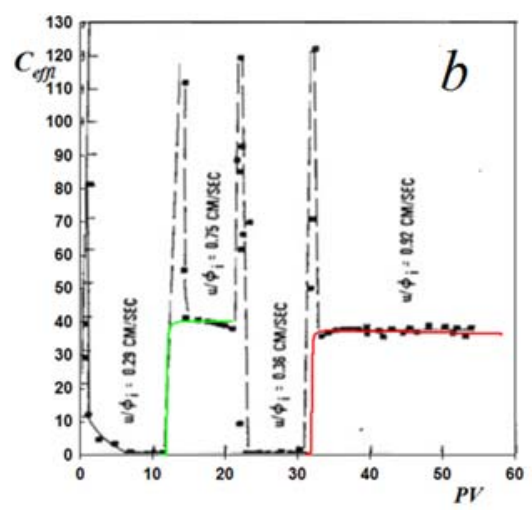

Figure 2: The concentration of suspended particles in the effluent $\left(10^{6}\right)$ against the number of pore volumes injected. Black curve - experiment, green curve - numerical simulations with fitted tuning parameters, red curve - numerical simulations with the same values of tuning parameters and the mobilization velocity. 
In order to compare the novel model against the classical one [2]-[5], the numerical simulations of experiments [14] are carried out. In these experiments, the suspension was injected into different "clean" porous samples and the profiles of trapped particle concentration was measured. The trapping coefficient $\lambda$ was tuned by minimization of the square-route deviation between the experimental and numerical particle concentration profiles. It was obtained that the new model provides better description of the trapped particle concentration in zones of a large particle accumulation close to a maximum value $\sigma=$ $C_{\max } \varphi_{0}$ (Fig. 3).

\section{RESULTS AND DISCUSSION}

In the framework of novel model (1)-(6), we carried out numerical simulations of suspension filtration in a porous medium representing the rock formation surrounding an injection well. The length of the porous medium is $1000 \mathrm{~m}$, the permeability is $k=10 \mathrm{mD}$, the porosity is $\varphi_{0}=0.1$. The results of simulations of contamination and cleanup of the porous medium is shown in Fig. 4(a). At the first stage, the flow of suspension is from left to right (injection) and particles can only be trapped. At the second stage, the flow is reversed from right to left (cleanup) and the particle-free fluid mobilizes the trapped particles. In Fig. 4(b) we show a simulation of the similar sequence, but with the velocity decreased from 1 to 0.1 $\mathrm{m} / \mathrm{s}$ and the trapping coefficient increased from 1 to $10 \mathrm{~m}^{-1}$. The profile of trapped particle concentration and permeability for these two cases are similar, while the penetration distances are different ( 5 and $0.5 \mathrm{~m}$, respectively). It is found that in both filtration conditions the permeability is almost completely restored, while the period of cleanup is significantly smaller than that of the injection.

We also simulated the injection and cleanup process taking into account both particle trapping and mobilization (Fig. 5). It is found that while the time of injection and production are the same, the permeability is not restored to its initial value. The study will be continued after the proper values of free parameters describing trapping and mobilization rates are found by tuning against proper experimental data.

\section{CONCLUSIONS}

The model of multiphase filtration of particle-laden fluids in porous media is developed. The number of tuning parameters of the model is decreased from four (typical to classical deepbed filtration model reported in the open literature) to two. The expression for critical mobilization velocity is established by considering the balance of forces acting on a single particle attached to the plane wall. The numerical solution of governing equations is carried out using a finite-difference approach.

The model is validated against a number of lab tests on contamination and cleanup of rock cores. It was obtained that the novel model provides better description of zones with large accumulation of trapped particles as compared to that obtained using the classical model with the same number of tuning parameters.

Numerical simulations of cyclic injection/cleanup regimes in a rock formation representing the surroundings of an injection well are carried out. The effects of particle trapping and mobilization on the dynamics of the rock permeability is preliminary studied. Further study requires the tuning of the suspension filtration model against the experimental data. 

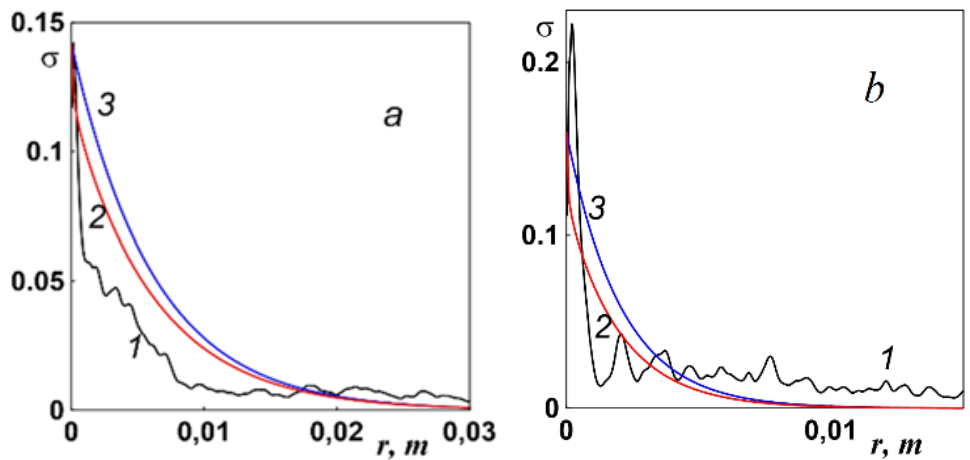

Figure 3: The concentration of trapped particles corresponding to the experiments of the injection of suspension in Bentheimer $(a)$ and Castlegate $(b)$ samples. Curve $1-$ the experiment, 2 - simulations using new model, 3 - classical model.
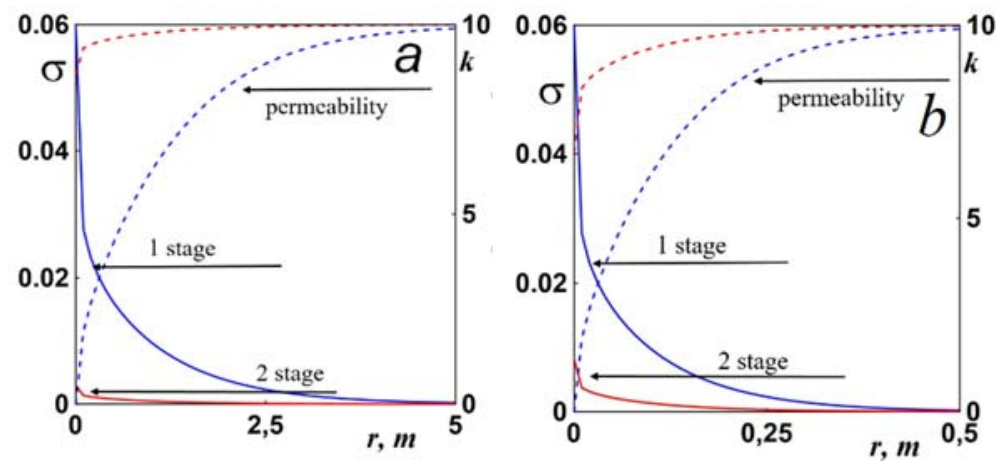

Figure 4: Profiles of trapped particle concentration (solid lines) and permeability (dashed lines) as a function of coordinate. Stage 1 is contamination with $\lambda=1 \mathrm{~m}^{-1}$, $t=60 \mathrm{~s}$, stage 2 is clean up with $\alpha=0.1 \mathrm{~m}^{-1}, t=30 \mathrm{~s}(a)$; stage 1 is contamination with $\lambda=10 \mathrm{~m}^{-1}, t=60 \mathrm{~s}$, stage 2 is clean up with $\alpha=1 \mathrm{~m}^{-1}, t=20 \mathrm{~s}(b)$.
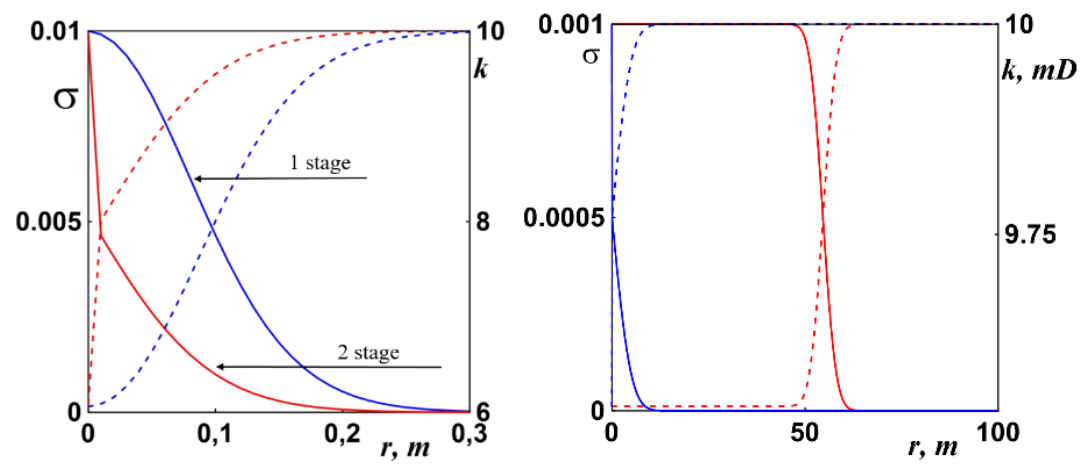

Figure 5: The trapped particle concentration (solid lines) and permeability (dashed lines) as a function of coordinate. Stage $1-\lambda=100 \mathrm{~m}^{-1}, \alpha=10 \mathrm{~m}^{-1}, t=10 \mathrm{~s}(\mathrm{a})$, $\lambda=10 \mathrm{~m}^{-1}, \alpha=10 \mathrm{~m}^{-1}, t=600 \mathrm{~s} \quad(b), \quad$ stage $2-\lambda=100 \mathrm{~m}^{-1}$, $\alpha=10 \mathrm{~m}^{-1}, t=10 \mathrm{~s}(a), \lambda=10 \mathrm{~m}^{-1}, \alpha=10 \mathrm{~m}^{-1}, t=600 \mathrm{~s}(b)$. 


\section{ACKNOWLEDGEMENT}

Startup funds of Skolkovo Institute of Science and Technology are gratefully acknowledged.

\section{REFERENCES}

[1] Civan, F., Reservoir Formation Damage, Gulf Professional Publishing, p. 1135, 2007.

[2] Herzig, J.P., Leclerc, D.M. \& Le Goff, P.L., Flow of suspensions through porous media - application to deep filtration. Industrial and Engineering Chemistry, 625, pp. 8-35, 1970.

[3] Barenblatt, G.I., Yentov, V.M. \& Ryzhik, V.M., The theory of the unsteady filtration of liquid and gas, NASA STI/Recon Technical Report N. 78, 1977.

[4] Boek, E.S., Hall, C. \& Tardy, P.M.J., Deep Bed Filtration Modelling of Formation Damage due to Particulate Invasion from Drilling Fluids. Transport in Porous Media, 91(2), pp. 479-508, 2012.

[5] Zamani, A. \& Maini, B., Flow of dispersed particles through porous media-deep bed filtration. Journal of Petroleum Science and Engineering, 691, pp. 71-88, 2009.

[6] Gruesbeck, C. \& Collins, R.E., Entrainment and deposition of fine particles in porous media. Society of Petroleum Engineers Journal, 22(6), pp. 847-856, 1982.

[7] Rajagopalan, R. \& Tien, C., Trajectory analysis of deep bed filtration with the sphere in-cell porous media model. AIChE Journal, 22(3), pp. 523-533, 1976.

[8] Boronin, S.A., Osiptsov, A.A. \& Tolmacheva, K.I., Multi-fluid model of suspension filtration in a porous medium. Fluid Dynamics, 50(6), pp. 759-768, 2015.

[9] Carman, P.C., Fluid flow through granular beds. Transactions, Institution of Chemical Engineers, 15, pp. 150-166, 1937.

[10] Scott, K.J., Hindered Settling of a Suspension of Spheres: Critical Evaluation of Equations Relating Settling Rate to Mean Particle Diameter and Suspension Concentration. Council for Scientific and Industrial Research, 1984.

[11] Zimon, A.D., Adhesion of dust and powder, Springer Science and Business Media, 2012.

[12] Brenner, H. \& Happel, J., Slow viscous flow past a sphere in a cylindrical tube. Journal of Fluid Mechanics, 4(2), pp. 195-213, 1958.

[13] Goldman, A.J., Cox, R.G. \& Brenner, H., Slow viscous motion of a sphere parallel to a plane wall. I Motion through a quiescent fluid. Chemical engineering science, 22(4), pp. 637-651, 1967.

[14] Mikhailov, D., Ryzhikov, N. \& Shako, V., An Integrated Experimental Approach to Determining How Invaded Mud Components Modify Near-Wellbore Properties (Russian). SPE Russian Oil and Gas Exploration \& Production Technical Conference and Exhibition, 2014. 